\title{
Experimental Study of Dibutyryl Cyclic AMP: Its Antishock Effects Observed in Traumatic Shock Rats
}

\author{
Ikuo Suemori and Junichi Yoshitake \\ Department of Anesthesiology, School of Medicine, Kagoshima \\ University, Kagoshima
}

\begin{abstract}
SUemori, I. and Yoshitake, J. Experimental Study of Dibutyryl Cyclic AMP: Its Antishock Effects Observed in Traumatic Shock Rats. Tohoku J. exp. Med., 1976, 119 (2), 123-133 — Antishock effect of $\mathrm{N}^{6}, \mathrm{O}^{2}$-dibutyryl eyclic AMP (DBcAMP) was investigated in rats subjected to Noble-Collip drum trauma and compared with effects of hydrocortisone and Trasylol. Results obtained are as follows. 1) Hydrocortisone and Trasylol administered $\mathrm{l} \mathrm{hr}$ before initiating drumming improved the survival rate from traumatic shock with concomitant reducton of levels of acid phosphatase and $\beta$-glucuronidase in circulating blood. DBcAMP administered i.p. immediately after trauma also improved the survival rate to the same extent as did Traylol or hydrocortisone, while no inhibitory effects were observed on acid phosphatase and $\beta$-glucuronidase. 2) The rectal temperature fell significantly after suffering trauma, and the rats with greater fall in rectal temperature had poorer chance for survival. The fall in rectal temperature was considerably prevented by DBcAMP in a dose of $0.5 \mathrm{mg} / 100 \mathrm{~g}$ body weight (b.w.). 3) DBcAMP induced a rise in plasma insulin level (IRI) and insulin/glucose ratio $(\mathrm{I} / \mathrm{G})$ in shock rats, and the elevation in blood lactate/ pyruvate ratio $(\mathrm{L} / \mathrm{P})$ and excess lactate otherwise observed after trauma were satisfactorily prevented by $\mathrm{DBCAMP}$ administration. It is concluded that the antishock effects of DBcAMP primarily resulted from improvements of the intracellular metabolism induced by its easy passage through the cell membrane and its cAMP like action, while any preventive action was not observed against elevation of lysosomal enzymes in the circulating blood. - DBcAMP; traumatic shock; antishock effect
\end{abstract}

A precarious state of circulation resulting from an overall insufficiency of blood flow connoted to the term "shock" leads to so serious hypoxia in various tissues that principal parts of biochemical abnormality found in the shock are ascribed to this hypoxia. Recently, Yoshitake (1975b) reported that the NAD/NADH ratios in the mitochondrial compartment of the rabbit liver cells shifted slightly to the oxidation side in the hemorrhagic shock. This suggests that the metabolic changes induced by shock result from relative deficiency of intramitochondrial substrates but not from hypoxia. He assumed that the metabolic defects in hemorrhagic shock had to be induced by the impairment of the substrate transport through the mitochondrial membrane. In a previous study, Suemori (1975)

Received for publication, December 23, 1975.

Presented at the Annual Meeting of the Kyushu Society of Surgery, June 14, 1975. 
investigated metabolic effects of DBcAMP administered to human subjects, and found that DBCAMP induced the elevations in blood glucose, serum insulin and pyruvate accompanied by a slight rise in blood lactate, consequently leading to a decrease in $\mathrm{L} / \mathbf{P}$ ratio and in excess lactate, and the increase in base excess. These results suggest that $D B C A M P$ could reverse metabolic alterations induced by shock. In this study, effects of DBeAMP on the survival rate, circulating lysosomal enzyme levels and metabolites levels in rats subjected to Noble-Collip drum trauma were investigated.

\section{Materials and Methods}

\section{Traumatic shock in the rats}

Male Wistar strain rats weighing about $200 \mathrm{~g}$ were subjected to rotational trauma in the Noble-Collip drum (1942). The challenging dose of trauma was 400 revolutions at $40 \mathrm{rpm}$, which caused $85 \%$ mortality in control rats within $4 \mathrm{hr}$ after the exposure. It was also confirmed that death only exceptionally occurred later than $4 \mathrm{hr}$ after drumming with this dose of trauma. In all experiments, rats were divided into pairs, one of which was taken as a control, and run at the same time in the paired drums of the shock machine. The rectal thermistor probe was used to monitor the core temperature of rats (Yellow Springs Instrument Co., Inc.).

1) The survival rates of shock rats were examined in the following groups; control ones, DBcAMP, ATP, hydrocortisone and Trasylol which were given $1 \mathrm{hr}$ before trauma or immediately after trauma.

2) Acid phosphatase and $\beta$-glucuronidase were measured in the circulating blood in each group. Rats were subjected to the sublethal dose of trauma (360 revolutions at 40 rpm) and blood was withdrawn from the abdominal aorta under light ether anesthesia. Effects of DBCAMP, hydrocortisone and Trasylol administered $1 \mathrm{hr}$ before drumming or immediately after drumming were compared.

3) Metabolic effects of DBeAMP were studied in the drum shock rats. The rats were subjected to the sublethal dose of drumming (360 revolutions at $40 \mathrm{rpm})$. Immediately after drumming, DBcAMP was administered in a dose of $0.5 \mathrm{mg} / 100 \mathrm{~g} \mathrm{b.w}$. i.p. and then blood was withdrawn at 30,60 or 120 min after drumming from abdominal aorta under light ether anesthesia for the determinations of hematocrit, serum protein, blood glucose, plasma insulin (IRI), blood lactate and pyruvate, plasma nonesterified fatty acids (NEFA), serum inorganic phosphorus and serum electrolytes. The methods for these determinations were described previously (Suemori 1975). Control determinations were performed with blood of rats without drumming.

\section{Plasma acid phosphatase assay}

The enzyme was assayed according to the method of Kind and King (1954) with reagents from IATRON Lab. Inc., Japan. Enzyme activity was expressed as millimoles of phenol released per hr at $37^{\circ} \mathrm{C}$ per $100 \mathrm{ml}$ of plasma.

\section{Plasma p-glucuronidase assay}

$\beta$-Glucuronidase was determined spectrophotometrically by measuring the $p$-nitrophenol isolated by decomposition of $p$-nitrophenyl glucuronide in an acidic solution ( $\mathrm{pH} 4.0$ ) at $400 \mathrm{~nm}$ using a spectrophotometer (Hitachi, Model 124). DBcAMP as sodium salt was supplied by Daiichi Pharmacentical Co. Ltd., Japan. 


\section{RESULTS}

Survival rates after trauma in control and treated rats

DBcAMP was administered intraperitoneally immediately after drumming, and additional doses were given $1-3$ times at $1 \mathrm{hr}$ intervals in some groups. The best result was obtained in the rats administered $0.5 \mathrm{mg} / 100 \mathrm{~g} \mathrm{b.w}$. of DBcAMP in a single dose immediately after trauma as can be seen in Table 1 . However, no favourable effect was observed with the same dose of DBcAMP given $1 \mathrm{hr}$ prior to drumming. Repeated administrations of DBcAMP $(0.5 \mathrm{mg} / 100 \mathrm{~g} \mathrm{~b}$.w.) at $1 \mathrm{hr}$ intervals were less effective. Survival rates of the rats were not improved at all with ATP (1 mg/100 g or $2 \mathrm{mg} / 100 \mathrm{~g}$ b.w., i.p.). Trasylol $(1,000 \mathrm{KIE} / 100 \mathrm{~g}$ b.w.) or hydrocortisone $(20 \mathrm{mg} / 100 \mathrm{~g} \mathrm{b.w.)} \mathrm{were} \mathrm{effective} \mathrm{in} \mathrm{improving} \mathrm{considerably} \mathrm{survival}$ rates, especially when administered prior to trauma.

TABLE 1. Effects of DBcAMP, ATP, Trasylol and hydrocortisone on the survival rate in drum shock rats

\begin{tabular}{|c|c|c|}
\hline \multirow{2}{*}{$\begin{array}{l}\text { Drug and dosage } \\
\text { administered/l00 g } \\
\text { body weight (I.P.) }\end{array}$} & \multicolumn{2}{|c|}{$\begin{array}{l}\text { Survival rate at } 4 \mathrm{hr} \\
\text { after drumming }(\%)\end{array}$} \\
\hline & $\begin{array}{l}\text { Administered } \\
\text { after drumming }\end{array}$ & $\begin{array}{r}\text { Administered } 1 \mathrm{hr} \\
\text { before drumming }\end{array}$ \\
\hline Control & 15 & 15 \\
\hline $\begin{array}{l}\text { DBcAMP } \\
\left.\begin{array}{l}0.2 \mathrm{mg} \times 1 \\
0.5 \mathrm{mg} \times 1 \\
0.5 \mathrm{mg} \times 2 \\
0.5 \mathrm{mg} \times 4 \\
1.0 \mathrm{mg} \times 4 \\
2.0 \mathrm{mg} \times 4\end{array}\right\} \text { hourly }\end{array}$ & $\begin{array}{r}60 \\
90 \\
70 \\
50 \\
10 \\
0\end{array}$ & 10 \\
\hline $\begin{array}{l}\text { ATP } \\
\qquad \begin{array}{l}1.0 \mathrm{mg} \times 1 \\
2.0 \mathrm{mg} \times 1\end{array}\end{array}$ & $\begin{array}{l}20 \\
20\end{array}$ & $\begin{array}{l}20 \\
20\end{array}$ \\
\hline $\begin{array}{l}\text { Trasylol } \\
\quad 1,000 \mathrm{KIE} \times 1\end{array}$ & 40 & 100 \\
\hline $\begin{array}{c}\text { Hydrocortisone } \\
20 \mathrm{mg} \times 1\end{array}$ & 50 & 90 \\
\hline
\end{tabular}

\section{Changes in the rectal temperature}

The changes in body temperature in rats subjected to trauma with or without DBcAMP administrations after trauma are illustrated in Fig. 1. The rectal temperature of rats fell rapidly following trauma. Temperatures below $35^{\circ} \mathrm{C}$ were frequently observed. In general, the rats responded to drumming with a greater temperature fall had poorer chance for survival. The fall of rectal temperature was fairly well prevented with a single dose of $0.5 \mathrm{mg} / 100 \mathrm{~g}$ b.w. of DBcAMP. However, with higher or repeated doses of DBcAMP, temperature tended to fall further than in control rats. Higher or repeated doses of DBCAMP looked to intensify the circulatory deterioration in shock rats, probably due to its systemic vasodilatating action. 


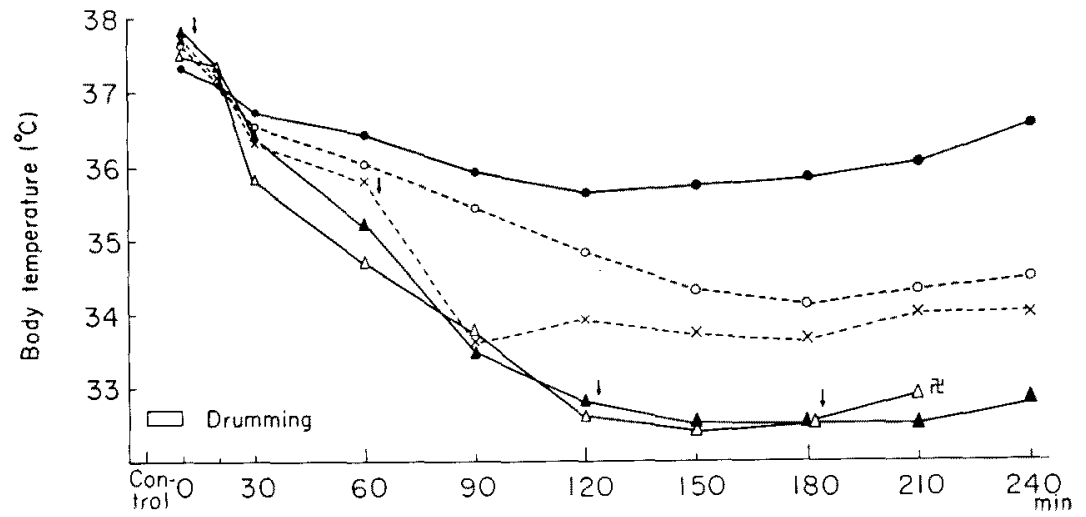

Fig. 1. Body temperature changes in rats administered with DBcAMP following Noble. Collip drumming (400 revolutions at $40 \mathrm{rpm}$ ). Each point indicates the average of

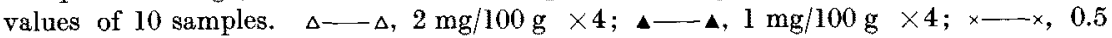
$\mathrm{mg} / 100 \mathrm{~g} \times 2 ; \bullet \ldots, 0.5 \mathrm{mg} / 100 \mathrm{~g} \times 1 ; 0 \ldots$. , without DBcAMP; $\downarrow$, DBcAMP administration.

The changes in plasma acid phosphatase and $\beta$-glucuronidase in rats during traumatic shock

The result are illustrated in Fig. 2. The levels of these enzymes increased progressively after trauma. Two hr after trauma, levels of plasma acid phosphatase were 3 times higher than the normal. At the same time, $\beta$-glucuronidase levels were also increased more than 4 times the normal in average. Thereafter no further increases in these enzymes levels were observed. Levels of acid phosphatase and $\beta$-glucuronidase $2 \mathrm{hr}$ after trauma in rats treated with Trasylol, hydrocortisone or DBcAMP $1 \mathrm{hr}$ before trauma are shown in Fig. 3 . Trasylol and hydrocortisone pretreatment did not significantly change the increase in plasma acid

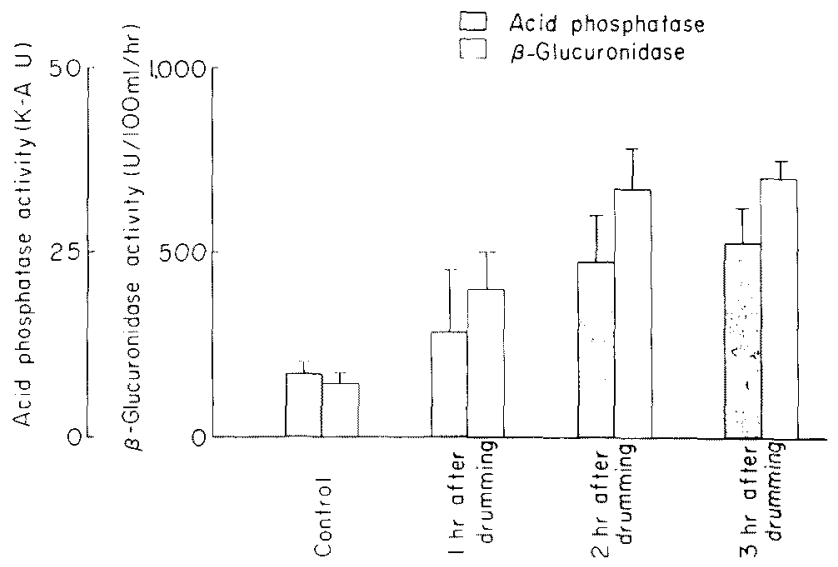

Fig. 2. Plasma acid phosphatase and $\beta$-glucuronidase activities after the drumming ( 360 revolutions at $40 \mathrm{rpm}$ ). 

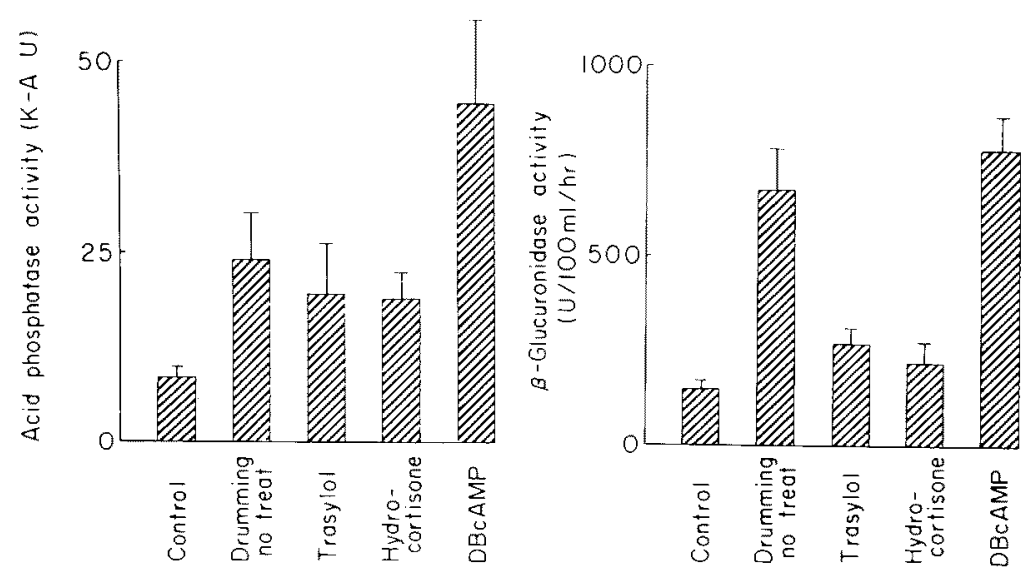

Fig. 3. Changes in plasma acid phosphatase and $\beta$-glucuronidase activities in drum shock rats. DBCAMP, Trasylol or hydrocortisone were administered $1 \mathrm{hr}$ before drumming.
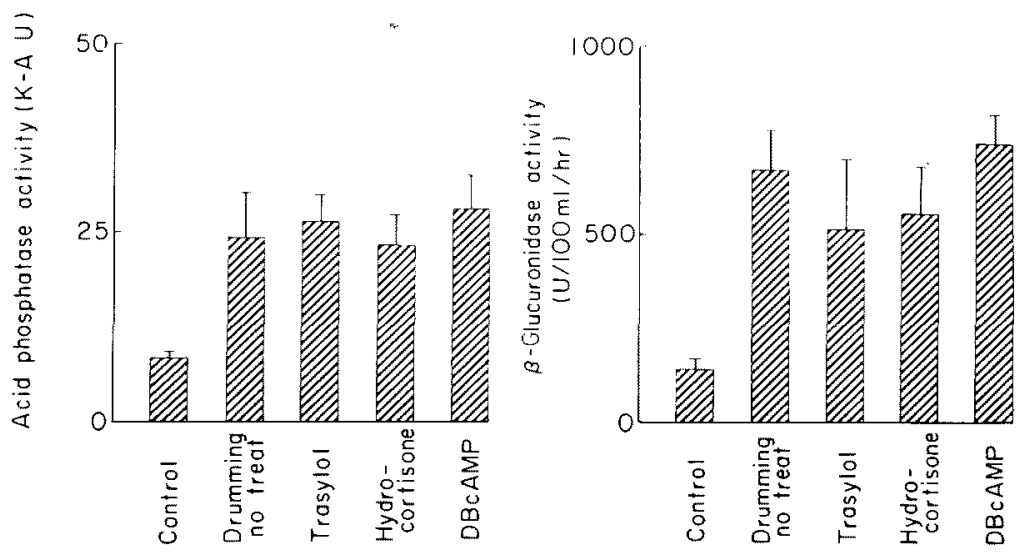

Fig. 4. Changes in plasma acid phosphatase and $\beta$-glucuronidase activities in drum shock rats. DBcAMP, Trasylol or hydrocortisone were administered after drumming.

phosphatase activity. On the other hand, they effectively prevented the increase in plasma $\beta$-glucuronidase activity. DBcAMP administered before trauma did not inhibit the increase in enzyme activity, but rather increased release of lysosomal enzymes. No significant inhibition was observed of increase in the activity of the plasma lysosomal enzyme by Trasylol, hydrocortisone and DBcAMP administration immediately after trauma as shown in Fig. 4. It was clearly demonstrated that the improvement of the survival rates in Trasylol- and hydrocortisone-pretreated rats was associated with an inhibition of plasma lysosomal enzyme activity.

Effects of DBcAMP on the metabolism of the drum shock rats

In order to elucidate the mechanism of the action of DBcAMP, which improved the survival rates of rats in traumatic shock, the metabolic effects of 

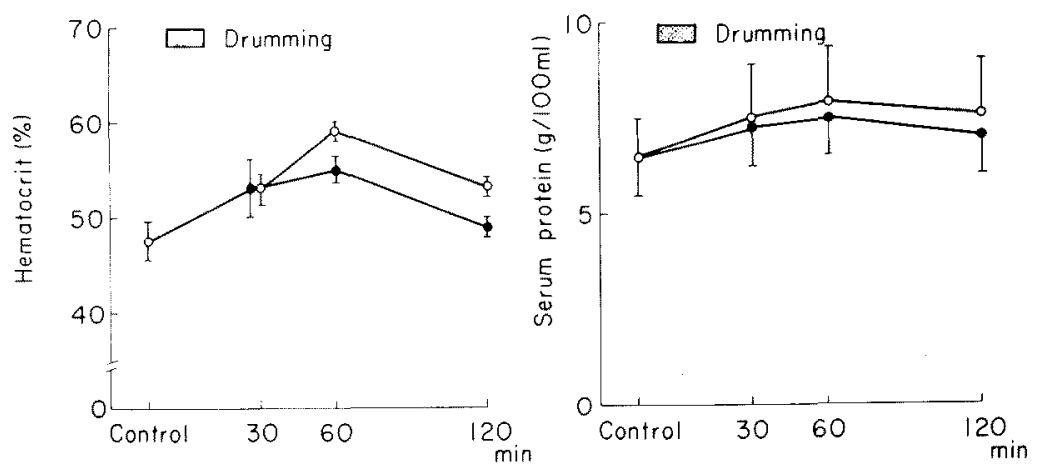

Fig. 5. Effects of DBCAMP on hematocrit and serum protein in drum shock rats. Each value is expressed as mean \pm s.e. $-\bullet$, with DBcAMP; ०-0, without DBcAMP.

DBcAMP on shock rats were further investigated. In this study, DBcAMP was administered in a dose of $0.5 \mathrm{mg} / 100 \mathrm{~g} \mathrm{b.w}$. immediately after drumming in sublethal dose. The hematocrit rose progressively until $1 \mathrm{hr}$ after drumming, then it declined slightly after $120 \mathrm{~min}$ in the untreated rats. There was no significant difference in hematocrit values between treated and untreated rats in $30 \mathrm{~min}$ after trauma, whereas significantly lower values were observed in the treated rats in 60 and $120 \mathrm{~min}$ after trauma as shown in Fig. 5. Serum protein levels also rose progressively during the first hour and slightly decreased by 120 min after drumming. In the rats treated with DBcAMP the changes in serum protein levels were smaller than those in the untreated rats (Fig. 5). From these results it is obvious that DBCAMP exerted a beneficial effect on usual hemoconcentration associated with this form of shock. In untreated rats a very pronounced fall was observed in blood glucose levels which reached almost one half the pretraumatic levels at 30 min after trauma, then it was followed by an increase to near pretraumatic values at 60 and 120 min after trauma. In DBcAMP treated rats the initial fall in blood glucose levels was of almost the same extent as that in the untreated rats. The depression of blood glucose level is followed by a significantly greater rise than that of the untreated in $1 \mathrm{hr}$ after drumming, then it reclined to the subnormal level in $120 \mathrm{~min}$ after trauma (Fig. 6). IRI fell to half the pretraumatic level in the untreated rats in $30 \mathrm{~min}$ after drumming, then it gradually returned to pretraumatic value. In the DBcAMP treated rats there was almost the same decrease in IRI as in the untreated rats in 30 min after drumming, which was followed by a steep rise in $60 \mathrm{~min}$ after trauma and then by restoration nearly to the pretraumatic level (Fig. 6). Consequently I/G ratios remained significantly higher in the DBcAMP-treated rats than those in untreated rats, which were lower than the pretraumatic value (Fig. 7). In the untreated rats a steep rise was observed in the serum levels of inorganic phosphorus, which reached almost 2 times the pretraumatic level within $30 \mathrm{~min}$ after trauma, and declined gradually to near pretraumatic level in $120 \mathrm{~min}$ after drumming. These increases in serum inorganic phosphorus induced by trauma were almost 

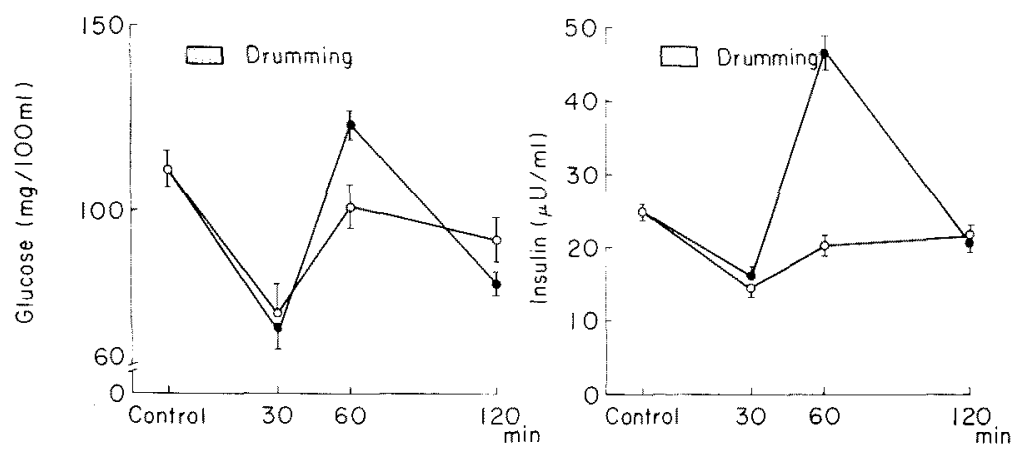

Fig. 6. Effects of DBcAMP on the blood glucose and plasma insulin in drum shock rats. Each value is expressed as mean \pm S.E. $-\bullet$, with DBcAMP; $0-0$, without DBcAMP.

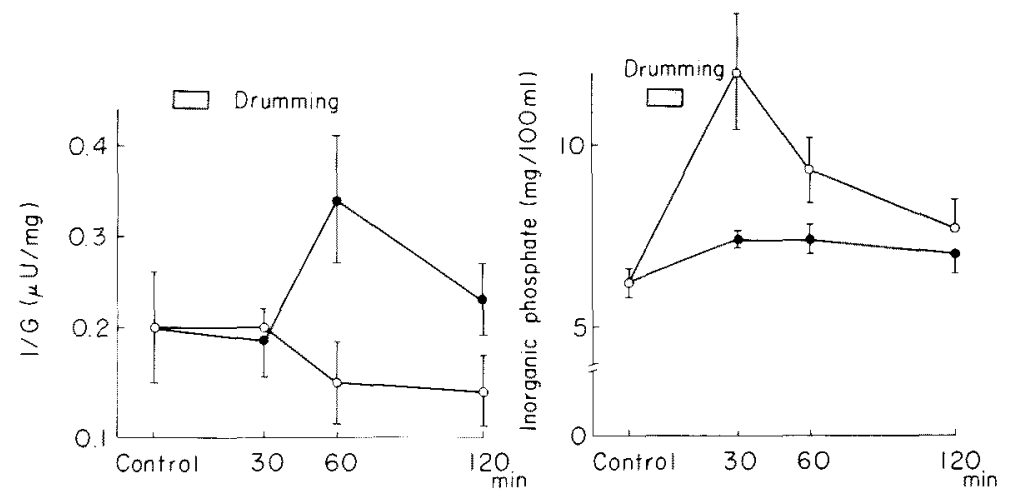

Fig. 7. Effects of DBcAMP on the $\mathrm{I} / \mathrm{G}$ ratio and inorganic phosphate in drum shock rats.

Each value is expressed as mean \pm s.t. - - with DBCAMP; O-o, without DBeAMP.

completely suppressed by DBcAMP treatment as illustrated in Fig. 7. The level of blood lactate was elevated progressively and reached a peak value of about 5 times higher than the pretraumatic value in $60 \mathrm{~min}$ after trauma, and then they returned to the pretraumatic level in $120 \mathrm{~min}$ after trauma. These increases in blood lactate were suppressed to some extent by DBcAMP treatment (Fig. 8). In the untreated rats, pronounced elevation in the level of blood pyruvate was observed $30 \mathrm{~min}$ after drumming. Blood pyruvate level returned to the pretraumatic value at $60 \mathrm{~min}$ after drumming. In the DBcAMP-treated rats blood pyruvate levels remained always higher than those in the untreated rats (Fig. 8). As a result, the $L / P$ ratio and excess lactate value elevated in the untreated rats, especially in $60 \mathrm{~min}$ after trauma, while those in the treated rats remained near pretraumatic levels as shown in Fig. 9. In the untreated rats plasma NEFA levels rose considerably after trauma, whereas those in the treated rats showed only slight elevation (Fig. 10). The serum $\mathrm{Na}$ and $\mathrm{K}$ concentrations remained unchanged after trauma in the DBcAMP treated rats. On the other hand, there was a considerable elevation in serum $\mathrm{K}$ in the untreated rats as shown in Fig. 11. 


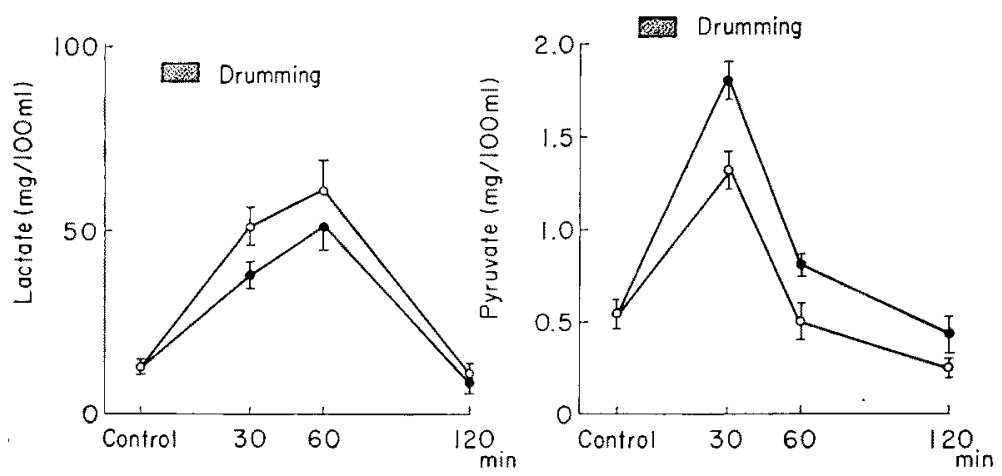

Fig. 8. Effects of DBcAMP on the blood lactate and pyruvate in drum shock rats. Each value is expressed as mean \pm S.E. - - $\bullet$ with DBCAMP; -0 , without DBcAMP.
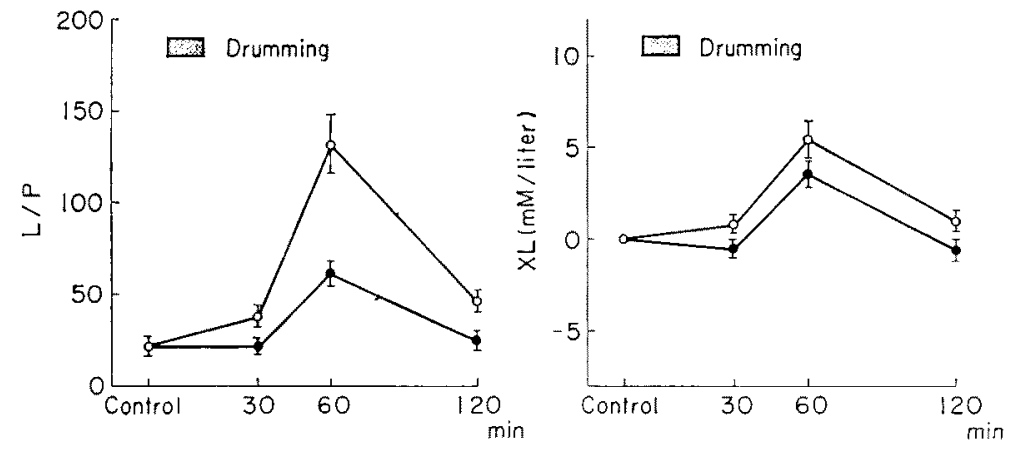

Fig. 9. Effects of DBeAMP on the $\mathrm{L} / \mathrm{P}$ ratio and excess lactate (XL) in drum shock rats. Each value is expressed as mean \pm S.E. - - $\bullet$, with DBcAMP; $0-0$, without DBcAMP.

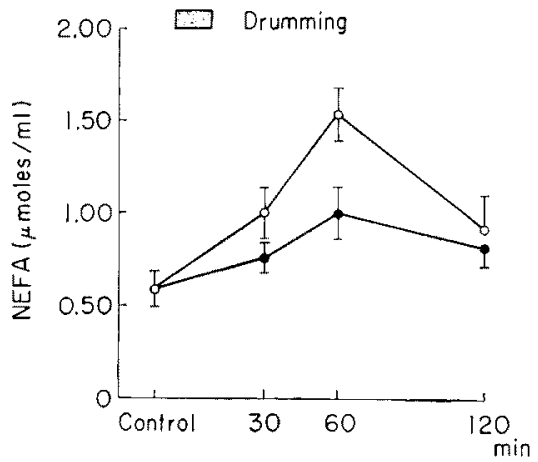

Fig. 10. Effects of DBcAMP on the plasma NEFA in drum shock rats. Each value is expressed as mean \pm s.E.

$\bullet-\bullet$ with DBeAMP; $-\circ$, without DBeAMP.

\section{Discussion}

DBcAMP, one of the analogue of cAMP, is supposed to penetrate biological membranes and to be more resistant to the phosphodiesterase. DBcAMP is 


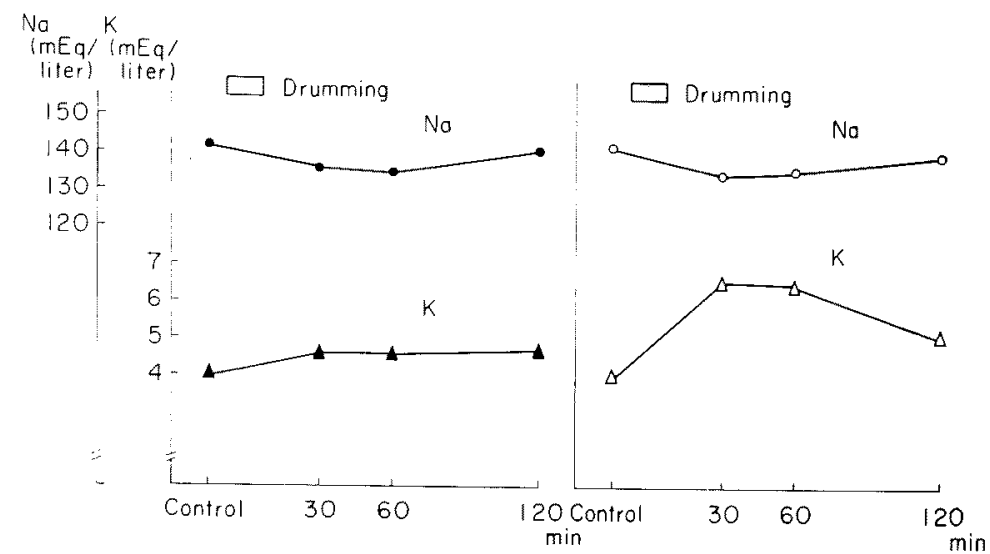

Fig. 11. Effects of DBcAMP on the plasma electrolytes in drum shock rats. Each value is expressed as mean. $\bullet, \mathbf{\Lambda}$, with DBcAMP;,$\Delta$, without DBcAMP.

converted to cAMP or biologically active $\mathrm{N}^{6}$-monobutyryl cAMP inside the cell. The survival rate of rats subjected to the Noble-Collip drum trauma was greatly improved with DBcAMP in the dose of $0.5 \mathrm{mg} / 100 \mathrm{~g}$. b.w. administered immediately after trauma probably by improvement of intracellular metabolism in various tissues. Lesser increases in survival following administration of repeated and higher doses of DBcAMP than $0.5 \mathrm{mg} / 100 \mathrm{~g}$ b.w. might be attributable to impairment of peripheral circulation being induced by vasodilating action of DBCAMP (Fredholm 1974). With an adequate measure of intravascular volume replacement, even higher doses of DBcAMP could exert a beneficial effect for survival of shock animals. Thus, DBcAMP was found to be protective for shock rats, whereas ATP was not. Therefore, the protective effects of DBcAMP was unlikely due to nonspecific effects of adenosine.

In this study, traumatic shock was consistently associated with an elevation of plasma levels of acid phosphatase and $\beta$-glucuronidase. The survival rate was remarkably improved following Trasylol and hydrocortisone pretreatments with concomitant depression of plasma levels of acid phosphatase and $\beta$-glucuronidase. These findings suggest that the appearance of these lysosomal enzymes in the circulation during shock might be considered as an index of the lethality of the shock state. DBcAMP administered immediately after trauma, however, did not depress the plasma levels of lysosomal enzymes in spite of improvement of survival rate. Therefore, the liberation of lysosomal enzymes by trauma might contribute as one of factors to the development of irreversible changes at the cellular levels during shock.

After trauma the hematocrit values invariably increased, indicating hemoconcentration (Chambers et al. 1943). The degree and duration of hemoconcentration therefore were of some value as an approximate indication of whether the rats would eventually survive.

It was also shown that trauma quickly lowered rat body temperature, if the 
environmental temperature was below the thermoneutral range. Body temperature below $35^{\circ} \mathrm{C}$ was frequently observed. Temperature fall had been found to be less reliable than changes in hemoconcentration as an indication of the severity of shock. Stoner (1969) reported that the fall in body temperature was due to decrease in heat production and $\mathrm{O}_{2}$ consumption. He found that the oxidation of injected pyruvate and palmitate was similarly inhibited after trauma, placing the metabolic "lesion" at or after the junction of these two main metabolic streams in the tricarboxylic acid cycle. Hemoconcentration induced by trauma was effectively prevented with DBcAMP. The increase in heat production seen in DBcAMP treated rats must mean that through the action of DBCAMP some oxidizable substrate(s) is reaching the TCA cycle.

Since energy production during shock is stated to shift to glycolysis, it is considered that the provision of exogenous glucose as an energy substrate might be of great improtance for survival. Veech et al. (1970) showed that a high glycolytic rate was associated with a high ATP and NAD in the cytosolic compartment, while gluconeogenesis was accompanied by a low ATP and NAD. In shock state the $\mathrm{NAD} / \mathrm{NADH}$ ratio in the cytoplasm shifted to reduction, which changed the equilibrium of the lactate dehydrogenase reaction in favor of lactate accumulation. The reduction in cellular ATP level was also shown in shock animals (Staples et al. 1969). As a result, exogenous glucose was little oxidized during shock as shown by Gump et al. (1973). DBcAMP produced a significant fall in $\mathrm{L} / \mathrm{P}$ ratio and in excess lactate in shock rats. This derivative is able to prevent the change in $\mathrm{NAD} / \mathrm{NADH}$ ratio brought about by shock. It was also reported that DBcAMP prevented the decrease in hepatic ATP levels in hemorrhagic shock rabbits (Yoshitake 1975a).

Concerning the reaction mechanism through which DBcAMP exerts its metabolic effects, Müllhofer et al. (1974) stated that DBcAMP enhanced flux of hydrogen equivalents from cytoplasm into mitochondria via "glycerol phosphate cycle". It is now accepted that "glycerol phosphate cycle" is more important for the transprot of reducing equivalents from cytoplasm into mitochondria than has been previously assumed.

Trauma as a stress stimulus activates the endocrine system which results in an increase of circulating catecholamine, glucocorticoids and ACTH (Bland 1956). Porte et al. (1966) has demonstrated that epinephrine can prevent a rise in plasma insulin despite the presence of the hyperglycemia. The mechanism of this effect is now known to involve inhibition of cAMP formation. There are clinical data which indicate insulin therapy is beneficial in patients suffering from shock following severe burn or myocardial infarction. In this study it was found that DBcAMP potentiated insulin release in shock rats. The insulin response observed did not appear to be secondary to DBCAMP-induced hyperglycemia.

Cyclic AMP has been considered as a second messenger mediating a variety of hormonal effects such as catecholamine, glucagon and ACTH at each target organ (Sutherland and Rall 1960). In shock state the enhanced activation in the endocrine system may induce the changes in the intracellular cAMP level. Yoshi- 
take (1975b) reported that the hepatic cAMP value was maintained well above the control value with rapid withdrawal of blood in 10 to $15 \mathrm{~min}$, whereas it was decreased somewhat when the animal was kept in hemorrhagic shock state for several hours. This means that the enhanced activity in the endocrine system induced by shock is not utlimately reflected in the modifications in the cellular functions and metabolism. In these situations DBcAMP, which is supposed to penetrate cell membrane readily and function like cAMP, must be a desirable agent for direct potentiation of cellular function and metabolism.

\section{Acknowledgment}

We were indebted to Dainchi Pharmaceutical Co. Ltd., Japan, for the supply of DBCAMP

The excellent technical assistance of Miss Teruko Sameshima and Miss Junko Miyao is gratefully acknowledged. We wish to express our gratitude to Prof. Koroku Hashimoto, Tohoku university, for many valuable suggestions and discussions on this work.

\section{References}

1) Bland, J.H. (1956) Clinical Recognition and Management of Disturbances of Body Fluids. Saunders Co., Philadelphia, U.S.A.

2) Chambers, R., Zweifach, B.W. \& Lowentein, B.E. (1943) Circulatory reactions of rats traumatized in the Noble-Collip drum. Amer. J. Physiol., 139, 123-128.

3) Fredholm, B.B. (1974) Vascular and metabolic effects of theophylline, dibutyryl cyclic AMP and dibutyryl cyclic GMP in canine subcutaneous adipose tissue in situ. Acta physiol. scand. 90, 226-236.

4) Gump, F.E., Long, C.L. \&. Wong, M. (1973) Exogenous glucose as an energy substrate in experimental hemorrhagic shock. Surg. Gynec. Obstet. 136, 611-617.

5) Kind, P.R.N. \& King, E.J. (1954) Estimation of plasma phosphatase by determination of hydrolysed phenol with amino-antipyrine. $J$. clin. Path. 7, 322-326.

6) Müllhofer, G., Loy, E., Wollenberg, P. \& Krämer, R. (1974) A possible role of the glycerol phosphate cycle in cyclic AMP-stimulated gluconeogenesis from lactate in perfused rat livers. Physiol. chem., 355, 239-254.

7) Noble, R.L. \& Collip, J.B. (1942) Quantitative method for the production of experimental traumatic shock without haemorrhage in unanaesthetized animals. Quart. J. exp. Physiol, 31, 187-200.

8) Porte, D., Graber, A.L., Kuzuya, T. \& Williams, R.H. (1966) The effect of epinephrine on immunoreactive insulin levels in man. J. clin. Invest., 45, 228-236.

9) Staples, D., Topuzlu, C. \& Blair, E. (1969) A comparison of adenosine triphosphate levels in hemorrhagic and endotoxic shock in the rat. Surgery, 66, 883-885.

10) Stoner, H.B. (1969) Studies on the mechanism of shock. The impairment of thermoregulation by trauma. Brit. J. exp. Path., 50, 124-138.

11) Suemori, 1. (1969) Experimental study of dibutyryl cyclic AMP; its metabolie effects observed in anaesthetized human subjects. Tohoku J. exp. Med., 117, 111-118.

12) Sutherland, E.W. \& Rall, T.W. (1960) The relation of adenosine $3^{\prime}, 5^{\prime}$-phosphate and phosphorylase to the actions of catecholamines and other hormones. Pharmacol. Rev., 12, 265-299.

13) Veech, R.L., Raijman, L. \& Krebs, H.A. (1970) Equilibrium relations between the cytoplasmic adenine nucleotide system and nicotinamide-adenine nucleotide system in rat liver. Biochem. J., 117, 499-503.

14) Yoshitake, J. (1975a) Metabolic aspects of shock. Saishin Igaku (Jap) 30, 17001707.

15) Yoshitake, J. (1975b) Metabolic disorder of shock. Igaku no Ayumi (Jap) 92. 602607. 